\title{
Reduction of gain depletion and saturation on a Brillouin-erbium fiber laser utilizing a Brillouin pump preamplification technique
}

\begin{abstract}
This paper presents the characteristics of a multiwavelength L-band Brillouin-erbium comb fiber laser with a preamplified Brillouin pump (BP) power technique at low pumping powers. The issue of erbium-doped fiber gain depletion and Brillouin gain saturation are resolved by the proposed structure. For long single-mode fiber length, the Stokes line emission occurs at low pumping powers because of the high strength of spontaneous Brillouin scattering, which provides a strong seed for coherent regenerative amplification of the Stokes line in the laser cavity. The laser structure achieves a low threshold power of $17 \mathrm{~mW}$ and is able to produce high number of output channels at low pumping powers. We experimentally show that the fiber laser structure can produce up to 37 channels at 55 and $0.045 \mathrm{~mW}$ of $1480 \mathrm{~nm}$ pump and BP powers, respectively.
\end{abstract}

Keyword: Optical amplifier; L-band; Gain-clamped; Brillouin-erbium; fiber laser; Brillouin pump (BP); Erbium-doped fiber gain depletion; Brillouin gain saturation 